Cinémas

Revue d'études cinématographiques

Journal of Film Studies

\title{
Index des livres
}

Volume 15, numéro 2-3, printemps 2005

Cinélekta 5

URI : https://id.erudit.org/iderudit/012334ar

Aller au sommaire du numéro

Éditeur(s)

Cinémas

ISSN

1181-6945 (imprimé)

1705-6500 (numérique)

Découvrir la revue

Citer ce document

(2005). Index des livres. Cinémas, 15(2-3), 255-258. d'utilisation que vous pouvez consulter en ligne.

https://apropos.erudit.org/fr/usagers/politique-dutilisation/ 


\section{Index des livres}

African Cinema: Politics and Culture (Manthia Diawara)

Vol. 4, n 2: 183

Alain Resnais. Une lecture topologique (Sarah Leperchey)

Vol. 14, n ${ }^{\text {os }} 2 / 3: 247$

Assia Djebar. Écrire, transgresser, résister (Jeanne-Marie Clerc)

Vol. 10, nos 2/3: 243

Au pays des ennemis du cinéma... Pour une nouvelle histoire des débuts du cinéma (André Gaudreault, Germain Lacasse, Jean-Pierre SiroisTrahan)

Vol. 8, $\mathrm{n}^{\text {os }} 1 / 2: 209$

Aventure américaine de l'ouvre de François Truffaut (L') (Laurence Alfonsi)

Vol. 12, n 3 : 175

Body in Film (The) (Bruce R. Elder) Vol. $7, \mathrm{n}^{\text {os }} 1 / 2: 245$

Bonimenteur de vues animées. Le cinéma " muet » entre tradition et modernité (Le) (Germain Lacasse) Vol. 12, $\mathrm{n}^{\circ}$ 3: 157

Canadian Catalogues and

Monographs of the Avant-garde, 1989-1995

Vol. $7, \mathrm{n}^{\text {os }} 1 / 2: 245$

Chicanos and Film. Representation and Resistance (Chon A. Noriega)

Vol. 4, n' 2: 177

Cinéma en histoire. Institution cinématographique, réception filmique et reconstitution historique (Le) (André Gaudreault, Germain Lacasse, Isabelle Raynault)

Vol. $10, n^{\text {os }} 2 / 3: 247$
Cinéma et culpabilité en Allemagne, 1945-1990 (Béatrice Fleury-Villatte) Vol. 8, ${ }^{\text {os }} 1 / 2: 203$

Cinéma français depuis 1945 (Le)

(René Prédal)

Vol. 4, n' 1: 151

Cinémas d'Afrique noire. Le regard en question (Les) (Olivier Barlet)

Vol. 8, $\mathrm{n}^{\circ}$ 3: 169

Corps au cinéma: Keaton, Bresson, Cassavetes (Le) (Vincent Amiel)

Vol. 11, nos 2/3: 309

Corps différé ou la force du regard au cinéma (Le) (Charles Perraton)

Vol. 11, n $1: 171$

Dark Alchemy. The Films of Jan

Svankmajer (Peter Hames)

Vol. 8, ${ }^{\text {os }} 1 / 2: 215$

David Rimmer: Films and Tapes

1967-1993 (Jim Shedden)

Vol. 7, $\mathrm{n}^{\mathrm{os}} 1 / 2: 245$

De beaux lendemains? Histoire, société et politique dans la sciencefiction (Gianni Hayer et Patrick J. Gyger)

Vol. 13, n $3: 193$

De la fiction (Roger Odin)

Vol. 12, n' 2: 187

Deleuze on Cinema (Ronald Bogue)

Vol. 14, n $1: 207$

Des mots à l'image. La voix over au cinéma (Jean Châteauvert)

Vol. 8, $n^{\circ}$ 3: 177

Dictionnaire de l'édition de cinéma (Jean-François Houben)

Vol. 13, $\mathrm{n}^{\text {os }} 1 / 2: 219$

Documentaire. Contestation et propagande (Le) (Catherine Saouter)

Vol. 8, nº 3: 161 
Écran de l'amour. Cinéma, érotisme et pornographie (L') (Martine Boyer) Vol. 2, $\mathrm{n}^{\text {os }} 2 / 3: 231$

Écran de la passion. Une étude du cinéma de Marguerite Duras (L') (Michelle Royer)

Vol. 8, n ${ }^{\circ} 3: 183$

Écraniques. Le film du texte (MarieClaire Ropars-Wuilleumier)

Vol. 3, nº 1: 149

Énonciation impersonnelle ou Le site du film (L') (Christian Metz)

Vol. 3, $\mathrm{n}^{\text {os }} 2 / 3: 241$

En route vers le parlant. Histoire

d'une évolution technologique,

économique et esthétique du cinéma (1926-1934) (Martin Barnier)

Vol. 14, ${ }^{\text {os }} 2 / 3: 255$

Épreuve du réel à l'écran. Essai sur le principe de réalité documentaire (L') (François Niney)

Vol. 12, $\mathrm{n}^{\circ}$ 3: 165

Espace au cinéma (L') (André Gardies)

Vol. $5, \mathrm{n}^{\text {os }} 1 / 2: 229$

Fabrications: Costume and the

Female Body (Jane Gaines et

Charlotte Herzog)

Vol. 3, n ${ }^{\text {os }} 2 / 3: 247$

Faire l'histoire du cinéma. Les

modèles américains (Robert C. Allen et Douglas Gomery)

Vol. 6, n ${ }^{\circ} 1: 171$

Film de famille. Usage privé, usage

public (Le) (Roger Odin)

Vol. 6, $\mathrm{n}^{\text {os }} 2 / 3: 223$

Filmusique, filmopéra (Maurice

Blackburn)

Vol. 6, $\mathrm{n}^{\text {os }} 2 / 3: 231$

Flashbacks in Film: Memory and

History (Maureen Turim)

Vol. 2, $\mathrm{n}^{\text {os }} 2 / 3: 223$

Fritz Lang (Lotte Eisner)

Vol. $9, \mathrm{n}^{\text {os }} 2 / 3: 237$
Fritz Lang en Amérique (Peter

Bogdanovich)

Vol. 9, $\mathrm{n}^{\text {os }}$ 2/3: 238

Fritz Lang: The Nature of the Beast.

A Biography (Patrick McGilligan)

Vol. 9, $\mathrm{n}^{\text {os }} 2 / 3: 240$

Fritz Lang. Trois lumières (Alfred

Abel)

Vol. 9, $\mathrm{n}^{\text {os }} 2 / 3: 236$

Générique de film (Le) (Nicole de

Mourgues)

Vol. 5, $\mathrm{n}^{\circ}$ 3: 187

Hauts et les bas de l'imaginaire

western (Les) (Paul Bleton et

Richard Saint-Germain)

Vol. 10, n 1: 167

Horreur intérieure: les films de

David Cronenberg (L') (Piers

Handling et Pierre Véronneau)

Vol. 2, $\mathrm{n}^{\text {os }}$ 2/3: 237

Images. De l'optique au numérique

(Edmond Couchot)

Vol. 1, n' 3: 146

Images de surface : l'art vidéo

reconsidéré (Christine Ross)

Vol. 9, n $1: 155$

Immédiateté. Anthropologie

culturelle critique (L') (Damien

François)

Vol. 12, n ${ }^{\circ} 1: 179$

Italian and Irish Filmmakers in

America: Ford, Capra, Coppola and Scorsese (Lee Lourdeaux)

Vol. 2, n $1: 170$

Itinéraire d'Alain Resnais (L') (René

Prédal)

Vol. 9, n $1: 159$

Lectures asiatiques de l'ouvre de

François Truffaut (Laurence Alfonsi)

Vol. 12, n 3: 175 
Limiti della rappresentazione (I). The Bounds of Representation. Censorship, the Visible, Modes of Representation in Film (Leonardo Quaresima, Alessandra Raengo, Laura Vichi)

Vol. 10, nos 2/3: 235

Making Meaning: Inference and

Rhetoric in the Interpretation of

Cinema

Vol. 1, $\mathrm{n}^{\text {os }} 1 / 2: 167$

Mémoire des déchets. Essais sur la culture et la valeur du passé (La)

(Claude Dionne, Brian Neville, Johanne Villeneuve)

Vol. 10, $\mathrm{n}^{\text {os }} 2 / 3: 227$

Montagne des superstitions et autres histoires (La) (Cornelius Schnauber) Vol. 9, $\mathrm{n}^{\text {os }}$ 2/3: 239

Mort d'un carriériste et autres histoires (Cornelius Schnauber)

Vol. 9, $\mathrm{n}^{\text {os }}$ 2/3: 239

Mystifying Movies : Fads and Fallacies in Contemporary Film Theory

Vol. 1, $\mathrm{n}^{\text {os }} 1 / 2: 155$

Narrative Comprehension and Film (Edward Branigan)

Vol. 4, n' 3: 177

New Vocabularies in Film

Semiotics: Structuralism, Poststructuralism, and Beyond (Robert Burgoyne, Sandy Flitterman-Lewis, Robert Stam)

Vol. 4, n 3: 167

Petite phénoménologie de l'écriture filmique (Lucie Roy)

Vol. 11, nos 2/3: 303

Pourquoi la fiction? (Jean-Marie Schaeffer)

Vol. 12, $\mathrm{n}^{\circ} 1: 169$
Pour une lecture sociocritique de l'adaptation cinématographique. Propositions méthodologiques (Monique Carcaud-Macaire et Jeanne-Marie Clerc) Vol. 7, $\mathrm{n}^{\text {os }} 1 / 2: 239$

Presence and Absence: The Films of Michael Snow, 1956-1991 (Jim Shedden)

Vol. 7, $\mathrm{n}^{\text {os }} 1 / 2: 245$

Rappresentazione dello sguardo nel cinema delle origini in Europa.

Nascita della soggettiva (La) (Elena

Dagrada)

Vol. 11, $\mathrm{n}^{\text {os }} 2 / 3: 321$

Récit cinématographique (Le)

(André Gaudreault et François Jost) Vol. 1, nº 3: 151

Récit filmique (Le) (André Gardies)

Vol. 5, $\mathrm{n}^{\text {os }} 1 / 2: 229$

Regard retourné. Aspects du cinéma des premiers temps (Le) (Livio

Belloï)

Vol. 13, $\mathrm{n}^{\text {os }} 1 / 2: 213$

Richard Hancox (Catherine

Jonasson)

Vol. 7, $\mathrm{n}^{\text {os }} 1 / 2: 245$

Richard Kerr: Overlapping Entries

(Cindy Richmond)

Vol. 7, $\mathrm{n}^{\text {os }} 1 / 2$ : 245

Secret du star system américain. Une stratégie du regard (Le) (Paul

Warren)

Vol. 1, $\mathrm{n}^{\text {os }} 1 / 2: 147$

Skin of the Film: Intercultural

Cinema, Embodiment, and the

Senses (The) (Laura U. Marks)

Vol. 11, nos 2/3: 293

Sound Theory. Sound Practice (Rick Altman)

Vol. 4, n ${ }^{\text {os }}$ 3: 157

Stanley Kubrick. A Narrative and

Stylistic Analysis (Mario Falsetto)

Vol. 6, $\mathrm{n}^{\text {os }} 2 / 3: 217$

Vol. 13, $\mathrm{n}^{\text {os }} 1 / 2: 229$ 
Subversive Pleasures. Bakhtine,

Cultural Criticism and Film (Robert Stam)

Vol. 2, $\mathrm{n}^{\circ} 1: 177$

Suisse, les Alliés et le cinéma (La)

(Gianni Haver)

Vol. 13, n $3: 193$

Temps d'un regard. Du spectateur

aux images (Le) (François Jost)

Vol. 10, $\mathrm{n}^{\circ} 1: 159$
Theater, Film, Literatur in

Frankreich. Medienwechsel und

Intermedialitat (Franz-Josef

Albersmeier)

Vol. 5, n ${ }^{\text {os }} 1 / 2: 223$

Un monde à notre image.

Énonciation, cinéma, télévision

(François Jost)

Vol. 5, $\mathrm{n}^{\circ}$ 3: 181 